\title{
Estudos de equivalência farmacêutica e perfil de dissolução comparativo de comprimidos contendo hidroclorotiazida
}

\author{
Pharmaceutical equivalence and dissolution profile studies for \\ tablets containing hydrochlorothiazide
}

Recebido em: 20/10/2017

Aceito em: $01 / 03 / 2018$

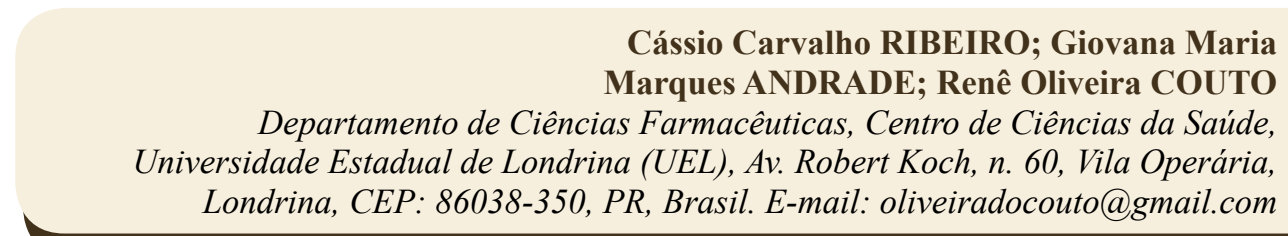

ABSTRACT

Hydrochlorothiazide (HCTZ) belongs to the thiazide diuretic class, widely used in the treatment of patients with mild to moderate edema and systemic arterial hypertension, being considered highly effective by health professionals. The purpose of this work was to evaluate the pharmaceutical equivalence and to perform comparative dissolution profile studies between the Reference medicine (R) and three Generic medicines from different brands (G1, G2 and G3) presented as immediate release tablets of hydrochlorothiazide $(25 \mathrm{mg})$. Such studies were carried out according to the monograph presented in the Brazilian Pharmacopoeia 5th Ed. (2010) and the current national legislation. In the physical and physicochemical tests, G1, G2 and G3 were approved. However, in some cases they presented significant differences when compared to R. Regarding the dissolution profile, which aims to evaluate the similarity factor (F2), only G3 did not present similar profile as compared to R. Thereby, interchangeability between G3 and R may be impaired once the drug may not reach the required plasmatic levels at the desired absorption rate and extension. Data arising from this study may reflect the quality and reliability of the generic medicines widely used from the Brazilian population.

Key-Words: hydrochlorthiazide; quality control; tablets; generic; dissolution

\section{RESUMO}

A hidroclorotiazida (HCTZ) é um fármaco da classe dos diuréticos tiazídicos, amplamente utilizado no tratamento de pacientes com edema e hipertensão arterial sistêmica leve a moderada, sendo considerado altamente efetivo pelos profissionais da saúde. O objetivo deste trabalho foi avaliar a equivalência farmacêutica e realizar estudos comparativos de perfil de dissolução entre medicamento Referência (R) e três medicamentos Genéricos (G1, G2 e G3) na forma de comprimidos de liberação imediata de HCTZ $(25 \mathrm{mg})$, de acordo com a monografia apresentada na Farmacopeia Brasileira $5^{\mathrm{a}}$ Ed. (2010) e a legislação nacional vigente. Nos testes físicos e físico-químicos todos os medicamentos $\mathrm{G}$ foram aprovados, no entanto, em alguns casos apresentaram diferenças significativas quando comparados ao medicamento R. Em relação ao perfil de dissolução, o qual visa avaliar o fator de semelhança F2, apenas o medicamento G3 não apresentou um perfil semelhante quando comparado ao medicamento R. A intercambialidade entre G3 e R pode estar comprometida, pois há possibilidade de não atingir níveis plasmáticos da HCTZ na velocidade e extensão desejadas. Os resultados deste trabalho podem refletir a qualidade e confiabilidade dos medicamentos genéricos amplamente utilizados pela população brasileira.

Palavras-chave: hidroclorotiazida; controle de qualidade; comprimidos; medicamentos genéricos; dissolução 


\section{INTRODUÇÃO}

Nas últimas décadas o mercado farmacêutico passou por diversas mudanças no que diz respeito ao modelo de negócio na comercialização de medicamentos, em especial no Brasil com a regulamentação da Lei Federal $n^{\circ} 6360 / 76$, alterada pela Lei $n^{\circ} 9787 / 99$, ou Lei dos Genéricos (1). A demanda crescente na produção de grandes indústrias tem relação direta com diversos fatores, como a diminuição dos custos de aquisição de medicamentos, a qualidade e o aumento da expectativa de vida da população mundial. Mesmo em tempos de crise, a indústria farmacêutica contemporânea mostra índices de crescimento elevados, com projeções bilionárias para os próximos anos. Estima-se que em 2020 o mercado farmacêutico global movimente US\$ 987 bilhões, valores que refletem um crescimento de $6,1 \%$ ao ano a partir de 2016 (2).

Estes fatos criam a necessidade do desenvolvimento de áreas pertinentes às Ciências Farmacêuticas, mais especificamente no controle da qualidade de medicamentos, um dos principais responsáveis pela inserção racional dos medicamentos Genéricos no mercado nacional, por meio de estudos que comprovem sua equivalência farmacêutica e bioequivalência em relação ao medicamento de Referência indicado pela Agência Nacional de Vigilância Sanitária (Anvisa) (3).

A intercambialidade entre os medicamentos Genéricos e de Referência tem sido amplamente discutida em publicações científicas (4-8). Storpirtis e cols. (2004) enfatizaram que as características físicas e físico-químicas do medicamento variam de acordo com a sua produção, e podem prejudicar a sua biodisponibilidade e bioequivalência (9). Porém, com o desenvolvimento farmacotécnico adequado, tal fato pode ser evitado, garantindo a sua qualidade e confiabilidade.

Formas farmacêuticas sólidas são as que mais sofrem impacto dos materiais e processos usados em sua produção, uma vez que quando comparadas às outras formas farmacêuticas como, por exemplo, líquidas, diversos fatores são relevantes quanto à interferência direta na dissolução do ativo, em sua disponibilidade, biodisponibilidade e bioequivalência. Os excipientes, processos, métodos e as próprias características inerentes ao fármaco são determinantes, e podem facilitar ou dificultar a sua liberação e absorção (9).

No Brasil, para a execução dos estudos de equivalência farmacêutica dos medicamentos genéricos em relação aos de referência, são adotados diversos testes físicos e físico-químicos comparativos, os quais são realizados por centros prestadores de serviços em equivalência farmacêutica devidamente habilitados pela Rede Brasileira de Laboratórios Analíticos em Saúde (REBLAS) (3). Sendo assim, é possível analisar cada medicamento, fabricante e forma farmacêutica de maneira individualizada, de modo que as particularidades de cada um sejam avaliadas, o que pode impactar diretamente na qualidade da produção de medicamentos em escala nacional e até global.

A hidroclorotiazida (HCTZ) é um diurético amplamente comercializado dentro do território nacional e um dos medicamentos mais distribuídos pelo Sistema Único de Saúde (SUS). Juntamente com os beta-bloqueadores, estes são os fármacos mais utilizados no tratamento de hipertensão nos últimos 40 anos, com eficácia comprovada na redução da morbidade e da mortalidade relacionadas à doença (10). Portanto, merece atenção especial no que diz respeito ao estudo da qualidade dos medicamentos produzidos nos diversos laboratórios em âmbito nacional e internacional, Genéricos, Similares ou de Referência, os quais podem causar grande impacto na saúde da população brasileira caso não forem efetivos.

A comparação das diversas marcas disponíveis no mercado é de suma importância e serve como base tanto para os profissionais de saúde, responsáveis pela prescrição e indicação desses medicamentos, quanto para a população de um modo geral, que representa o consumidor final do produto e necessita também de informações aprofundadas e confiáveis a respeito das possibilidades e alternativas terapêuticas disponíveis para o tratamento de suas doenças. Diante desse contexto, o objetivo deste trabalho foi realizar estudos de equivalência farmacêutica e perfil de dissolução comparativo de diversas marcas de medicamentos contendo HCTZ.

\section{MATERIAL E MÉTODOS}

Material. A HCTZ Substância Química de Referência (SQR, 99,9\%), utilizada em todos os ensaios quantitativos, foi gentilmente fornecida pela Sandoz Brasil Indústria Farmacêutica (Cambé, Paraná, Brasil). Foram analisadas quatro marcas diferentes de comprimidos contendo $25 \mathrm{mg}$ de HCTZ, sendo um produto de Referência (R) e três genéricos (G1, G2 e G3), adquiridos em drogarias do município de Londrina - PR. Cada amostra foi composta de exemplares do mesmo lote. 
Equipamentos. Foram utilizados lavadora ultrassônica USC-3300 (Unique, Barueri, SP, Brazil), balança analítica AdventurTM (Ohaus ${ }^{\circledR}$, Barueri, SP, Brasil); aparelho de dissolução DT800 (ERWEKA ${ }^{\circledR}$, Heusenstamm, Alemanha); espectrofotômetro UV 1800 (SHIMADZU $^{\circledR}$ do Brasil, Barueri, SP, Brasil); durômetro digital TBH` 125 (ERWEKA ${ }^{\circledR}$, Heusenstamm, Alemanha); friabilômetro Micron ${ }^{\circledR}\left(\right.$ ERWEKA $^{\circledR}$, Heusenstamm, Alemanha); e aparelho de desintegração VanKel ${ }^{\circledR}$ VK 100 (Boston Lab Co, Woburn, MA, EUA). Todos os equipamentos utilizados foram devidamente qualificados e calibrados antes dos ensaios.

Estudos de equivalência farmacêutica. Seguiram as diretrizes propostas pela legislação vigente no Brasil (3). Foram realizados testes físicos e físico-químicos de acordo com os métodos gerais descritos na Farmacopeia Brasileira $5^{\mathrm{a}}$ ed. (11) e monografia para comprimidos de HCTZ neste mesmo compêndio (12).

Determinação de peso médio. Vinte comprimidos foram individual e aleatoriamente pesados em balança analítica. Foram calculados o peso médio e o coeficiente de variação ou Desvio Padrão Relativo (DPR, \%) das médias (11).

Teste de dureza. Foi verificada em dez comprimidos, utilizando-se durômetro digital. A força requerida para ruptura radial dos comprimidos foi medida em Newton $(\mathrm{N})$, sendo expressa como média (DPR, \%) (11).

Teste de friabilidade. Foram pesados inicialmente 20 comprimidos de cada amostra. Em seguida, estes foram colocados no friabilômetro e retirados depois de efetuadas 100 rotações durante 4 minutos ( $25 \mathrm{rpm}$ ). Foi removido qualquer resíduo de pó da superfície dos comprimidos e os mesmos foram novamente pesados (11). A percentagem de perda de massa foi calculada segundo a equação [1]:

$$
\text { Friabilidade }=\frac{(\text { Peso inicial }- \text { Peso final })}{\text { Peso inicial }} \times 100, \%
$$

\section{[1]}

Teste de desintegração. Para cada amostra, 6 comprimidos foram colocados em aparato desintegrador, utilizando-se $900 \mathrm{~mL}$ de solução de $\mathrm{HCl}$ 0,1 M mantida a $37 \pm 1{ }^{\circ} \mathrm{C}$ como meio de desintegração. Foi registrado o tempo para que os comprimidos se desintegrassem completamente (11).

Doseamento. Vinte comprimidos de cada uma das apresentações foram pesados e pulverizados. Uma quantidade de pó equivalente a $30 \mathrm{mg}$ de HCTZ foi trans- ferida para balão volumétrico de $50 \mathrm{~mL}$ e dissolvida com quantidade suficiente de $\mathrm{NaOH} 0,1 \mathrm{M}$, com auxílio de banho de ultrassom, por 20 minutos. $\mathrm{O}$ volume foi completado com o mesmo solvente, e filtrado. Foram transferidos $0,5 \mathrm{~mL}$ do filtrado para balão volumétrico de $10 \mathrm{~mL}$, e o volume completado com $\mathrm{NaOH} 0,1 \mathrm{M}$. As absorbâncias das soluções correspondentes às amostras e HCTZ SQR preparada na mesma concentração foram verificadas em $273 \mathrm{~nm}$, utilizando $\mathrm{NaOH} 0,1 \mathrm{M}$ para ajuste do zero (12). Os experimentos foram realizados em triplicata, sendo os resultados expressos como média (DPR, \%).

Uniformidade de doses unitárias (UDU). Foi determinada pelo método de uniformidade de conteúdo (11). Foram pesados, exatamente e individualmente, 10 comprimidos. Empregando o mesmo método de doseamento, foi estimada a quantidade de HCTZ em cada unidade e expressos os resultados individuais em porcentagem da quantidade declarada. Os teores individuais estimados ( $x i)$ foram calculadas segundo a equação [2]:

$$
x i=\frac{(A b s A \times P P d \times P M A)}{(A b s P d \times P A \times D T)} \times 100, \quad \% \text { [2] }
$$

Onde: $A b s A$, absorbância da amostra, $P P d$, massa do padrão, $P M A$, peso médio da amostra, $A b s P d$, absorbância do padrão, $P A$, massa individual de cada comprimido e $D T$, dose declarada do medicamento.

Os Valores de Aceitação (VA) foram determinados de acordo com a Farmacopeia Brasileira $5^{\text {a }}$ Ed. (11). Foram considerados os casos 1a $(T \leq 101,5 ; 98,5 \leq m \leq$ $101,5 ; \mathrm{M}=m), 1 \mathrm{~b}(T \leq 101,5 ; m \leq 98,5 ; \mathrm{M}=98,5)$ e $1 \mathrm{c}$ $(T \leq 101,5 ; m>101.5 ; \mathrm{M}=101.5)$ em que $T$ representa a média dos limites especificados na monografia individual da HCTZ para a quantidade ou potência declarada, expressa em porcentagem, $m$ a média dos conteúdos individuais da quantidade declarada, também expressa em porcentagem, $n$ o número de unidades testadas, $k$ a constante de aceitabilidade e $s$ o desvio padrão da amostra. Os VA foram calculados usando as equações [3] a [5], respectivamente:

$$
\begin{gathered}
V A=(k * s) \quad[3] \\
V A=[98,5-m]+(k * s) \\
V A=[m-101.5]+(k * s)
\end{gathered}
$$

Foi considerado L1 o valor máximo permitido para o valor de aceitação, sendo 15,0. O número de comprimidos (n) utilizados foi de 10 unidades, portanto o valor de $k=2,4$. 
Teste de dissolução. Foi realizado com 6 comprimidos de cada amostra. Os comprimidos foram colocados aleatória e separadamente em cubas do dissolutor, contendo como meio de dissolução $900 \mathrm{~mL}$ de $\mathrm{HCl} 0,1 \mathrm{M}$ na temperatura de $37 \pm 0,5^{\circ} \mathrm{C}$. Como aparato de dissolução foram utilizadas cestas (aparato I), ajustadas a $100 \mathrm{rpm}$ por $30 \mathrm{~min}$, e posteriormente foram coletadas uma amostra de cada cuba de dissolução para análise.

As absorbâncias das amostras foram medidas em $272 \mathrm{~nm}$, utilizando o mesmo solvente para ajuste do zero. As quantidades de HCTZ dissolvidas no meio foram calculadas comparando as leituras obtidas para as amostras e HCTZ SQR na mesma concentração, preparada em $\mathrm{HCl} 0,1 \mathrm{M}(12)$.

Estudo de perfil de dissolução comparativa. Foram utilizados os mesmos parâmetros relativos ao teste de dissolução. Em tempos pré-determinados (i.e., 1, 5, $10,15,20,25$ e $30 \mathrm{~min}$ ), amostras de $20 \mathrm{~mL}$ do meio de dissolução foram retiradas, sendo o volume reposto com volume idêntico do mesmo meio. As amostras foram filtradas e diluídas apropriadamente com $\mathrm{HCl} 0,1 \mathrm{M}$. As absorbâncias das amostras foram medidas em $272 \mathrm{~nm}$, utilizando o mesmo solvente para ajuste do zero. A cada amostragem, as quantidades de HCTZ dissolvidas no meio foram calculadas comparando as leituras obtidas para as amostras e HCTZ SQR na mesma concentração, preparada em $\mathrm{HCl} 0,1 \mathrm{M}$ (12). Para comparar os perfis de dissolução dos medicamentos G1, G2 e G3, em relação ao $\mathrm{R}$, foram calculados, a partir das cedências médias ( $\mathrm{n}$ $=12$ ) em cada intervalo de tempo, o fator de similaridade (F2), segundo a equação [6], como proposto na RDC $31 / 2010$ (3):

$$
F 2=50 \times \log \left\{\left[1+\left(\frac{1}{n}\right) \sum_{t=1}^{n}(R t-T t)^{2}\right]^{-0,5} \times 100\right\}
$$

\section{[6]}

Onde: $\mathrm{n}$, número de tempos de coleta considerados para fins de cálculo de F2; Rt, valor de porcentagem dissolvida no tempo t, obtido com o Medicamento de Referência ou Comparador; $\mathrm{Tt}$, valor de porcentagem dissolvida do Medicamento Teste ou da formulação alterada, no tempo $t$.

Análise estatística. As médias obtidas para os medicamentos G1 - G3 foram comparadas com as do medicamento $\mathrm{R}$ aplicando Teste-t de Student pareado, utilizando o software GraphPad Instat 5.0 (GraphPad Software Inc., CA, EUA). As análises de regressão e correlação linear pelo método dos míni- mos quadrados foram realizadas utilizando o mesmo software. Em todas as análises, foram considerados significativos $p \leq 0,05$.

\section{RESULTADOS E DISCUSSÃO}

A Tabela 1 mostra os resultados obtidos nos testes realizados no estudo de equivalência farmacêutica. Nos testes de peso médio, os comprimidos R e G2 apresentaram resultados semelhantes, assim como G1 e G3, sendo que $\mathrm{R}$ apresentou menor peso médio $(109,88 \mathrm{mg})$ e o genérico G3 apresentou o maior peso médio $(127,06 \mathrm{mg})$, fato que pode indicar uma menor quantidade de excipientes por parte do medicamento R. No que diz respeito ao desvio padrão, o medicamento $\mathrm{R}$ atingiu o maior valor, chegando a 2,24, seguido pelos genéricos G3, G1 e G2, com valores de 2,08, 1,44 e 1,26 respectivamente. Segundo os critérios da Farmacopeia Brasileira $5^{\mathrm{a}}$ ed. (11), para comprimidos são tolerados não mais que duas unidades fora dos limites especificados a seguir, porém nenhuma unidade poderá estar acima ou abaixo do dobro da porcentagem indicada: $80 \mathrm{mg} \leq$ massa $\leq 250 \mathrm{mg}$, $\pm 7,5 \%$ de variação. Portanto, todos os medicamentos cumpriram o teste.

A variação de massa dos comprimidos no teste deve ser mínima para que padrões de qualidade sejam atingidos, pois assim é possível garantir que uma quantidade adequada de fármaco está presente na formulação. Caso este atributo de qualidade não esteja de acordo com o especificado, a eficácia terapêutica do medicamento pode ser comprometida, não produzindo a ação desejada ou acentuar os efeitos colaterais em função de uma sobredose medicamentosa. (13)

Nos testes de dureza, os comprimidos mostraram-se bastante diferentes em relação à média de seus valores. O medicamento $\mathrm{R}$ novamente apresentou menor valor médio $(39,9 \mathrm{~N})$ com um desvio padrão de 3,51, e o genérico G3 apresentou o maior valor médio $(60,7 \mathrm{~N})$ com um desvio padrão 4,85. Nos testes de friabilidade todos os medicamentos foram aprovados, ou seja, não atingiram valores maiores do que $1,5 \%$, como é preconizado pela Farmacopeia Brasileira (11), sendo G2 o comprimido menos friável $(0,10 \%)$ e G1 o que atingiu o maior valor de friabilidade $(0,17 \%)$.

Para suportarem choques mecânicos durante a produção, embalagem, transporte e manuseamento do paciente, os comprimidos devem apresentar dureza e friabilidade adequadas. Além disso, um comprimido muito 
duro pode interferir diretamente na sua desintegração e velocidade de dissolução do fármaco, fator que pode prejudicar o paciente e novamente interferir na eficácia terapêutica do medicamento (13).

Em todas as formulações os excipientes utilizados mostraram ser bastante semelhantes, a grande maioria com função de diluente e um lubrificante em comum, o estearato de magnésio (14), que por possuir propriedades hidrofóbicas pode aumentar o tempo de desintegra- ção quando não empregado na formulação de maneira adequada.

Os outros excipientes utilizados incluem lactose monoidratada, amido de milho, amido pré-gelatinizado e celulose microcristalina, todos com função diluente, e em alguns casos função desintegrante e aglutinante como no composto pré-gelatinizado. Os diluentes têm por função o aumento no volume dos pós e tornam viáveis o processo de compressão direta.

Tabela 1. Resultados de peso médio, dureza, friabilidade, tempo de desintegração, teor e uniformidade de dose unitária no estudo de equivalência farmacêutica entre medicamentos Referência (R) e Genéricos (G) contendo HCTZ (25 mg)

\begin{tabular}{ccccccc} 
Medicamento & PM (DPR, \%) & $\begin{array}{c}\text { D (N } \\
\text { (DPR, \%) }\end{array}$ & F (\%) & TD (min) & $\begin{array}{c}\text { THCTZ (\%) } \\
(\text { DPR, \%) }\end{array}$ & UC - VA \\
\hline R & $109,88(2,24)$ & $39,9(3,51)$ & 0,12 & $<2$ & $96,01(2,65)$ & 8,72 \\
G1 & $128,65(1,26)^{* * *}$ & $54,7(5,85)^{* * *}$ & 0,17 & $<15$ & $95,38(1,45)$ & 3,51 \\
G2 & $110,77(1,44)$ & $40,3(3,02)$ & 0,10 & 2 & $96,95(1,46)$ & 5,57 \\
G3 & $127,06(2,08)^{* * *}$ & $60,7(4,85)^{* * *}$ & 0,12 & $<9$ & $98,94(0,09)$ & 6,64
\end{tabular}

Diferença estatisticamente significativa quando comparado ao Medicamento Referência $-\mathrm{R}\left({ }^{*} p<0,05 ;{ }^{* *} p<0,01 \mathrm{e} * * * p<0,001\right.$; Teste $\mathrm{t}$ de Student); PM, peso médio $(\mathrm{mg}) ; \mathrm{D}$, dureza $(\mathrm{N}) ; \mathrm{F}$, friabilidade $(\%) ; \mathrm{TD}$, tempo de desintegração (min); THCTZ, teor de HCTZ (\%); UC, uniformidade de conteúdo, VA, valor de aceitação; DPR, desvio padrão relativo (\%).

Tabela 2. Resultados do teste de dissolução de HCTZ $(25 \mathrm{mg})$ no estudo de equivalência farmacêutica entre medicamentos Referência (R) e Genéricos (G)

\begin{tabular}{|c|c|c|c|c|c|}
\hline Medicamento & Estágio & $\begin{array}{c}\text { Maior Teor } \\
\text { Dissolvido (\%) }\end{array}$ & $\begin{array}{c}\text { Menor Teor } \\
\text { Dissolvido }(\%)\end{array}$ & $\begin{array}{c}\text { Média } \\
\text { Dissolvida (\%) }\end{array}$ & DPR (\%) \\
\hline $\mathrm{R}$ & E1 & 93,37 & 89,12 & 92,27 & 1,59 \\
\hline G1 & E1 & 98,05 & 91,61 & 95,00 & 2,99 \\
\hline G2 & E1 & 100,39 & 92,93 & 96,32 & 3,16 \\
\hline G3 & E1 & 95,12 & 90,29 & 92,71 & 1,84 \\
\hline
\end{tabular}

$\mathrm{n}=6$; aparato I (cestas); $900 \mathrm{~mL} \mathrm{HCl} \mathrm{0,1M;} 37 \pm 0,5^{\circ} \mathrm{C} ; 100 \mathrm{rpm} ; 30$ min.; DPR, desvio padrão relativo (\%).

Além disso, possibilitam alcançar o tamanho desejado do comprimido, uma vez que a dose utilizada do ativo em alguns comprimidos, como o da HCTZ, é muito pequena. Os desintegrantes são responsáveis por promover a ruptura dos comprimidos quando estes entram em contato com o meio fisiológico, acelerando o processo de desintegração da forma farmacêutica e dissolução do fármaco (15).

Diante disto, pode ser observada a correlação entre os pesos médios dos comprimidos e suas durezas, uma vez que não foi observada diferença significativa na escolha dos excipientes para a produção. A utilização de amido de milho e amido pré-gelatinizado como diluentes nas formulações justifica a diferença entre os valores, pois o uso destes excipientes aumenta a dureza dos comprimidos (14), fato o qual possibilita inferir que uma maior proporção de excipiente presente na formulação resulta, consequentemente, em um comprimido mais duro, como mostrado na Figura 1.

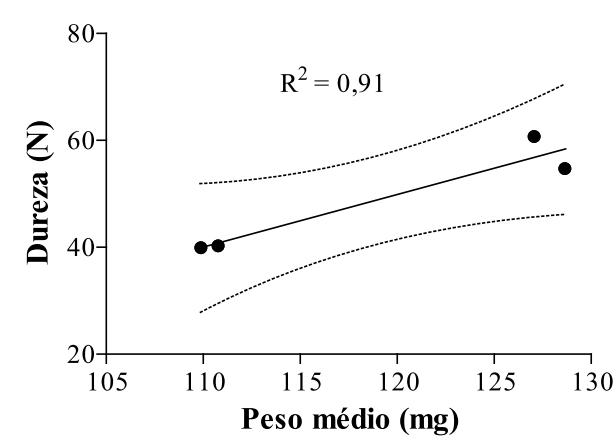

Figura 1. Correlação entre dureza e peso médio de comprimidos contendo HCTZ $(25 \mathrm{mg})$ para medicamentos Referência e Genéricos

No teste de desintegração, a Farmacopeia Brasileira $5^{\mathrm{a}}$ ed. (11), estabelece que o limite de tempo para que este seja cumprido é de 30 minutos. Novamente todos os comprimidos foram aprovados, com tempos respectivos para R, G1, G2 e G3 de 1min59s, 14min33s, 2min e 8 min57s. Antes do fármaco ser absorvido pelo orga- 
nismo, é necessário, para a maioria dos comprimidos, dependendo do seu mecanismo de liberação, que se desintegre, possibilitando a solubilização do ativo em meio fisiológico (13).

Portanto, quanto mais rápido for esse processo, mais rápido o fármaco entrará em contato com o meio, o que resultará em diferenças em relação ao tempo de dissolução e absorção, como foi confirmado nos testes de perfil de dissolução, em que G1 e G3, por possuírem maiores tempos de desintegração, encontram-se menos dissolvidos no primeiro intervalo de tempo.

A partir dos resultados foi possível também estabelecer uma correlação positiva moderada entre a dureza e o tempo de desintegração dos comprimidos, ou seja, quanto maior a dureza, maior o tempo de desintegração (Figura 2).

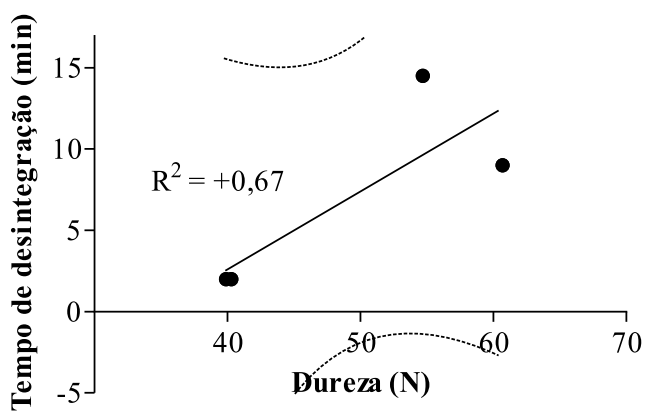

Figura 2. Correlação entre dureza e tempo de desintegração de comprimidos contendo HCTZ (25 mg) para medicamentos Referência e Genéricos

Para o teste de teor da HCTZ, a Farmacopeia Brasileira $5^{\text {a }} \mathrm{Ed}(12)$ preconiza que os comprimidos tenham valores entre no mínimo $93 \%$ e no máximo $107 \%$ da quantidade declarada do ativo. Sendo assim, novamente todos os produtos analisados apresentaram valores dentro dos limites especificados. O genérico G3 alcançou a maior média quando comparado aos outros medicamentos $(98,94 \%)$ e o genérico G1 apresentou menor média de valores $(95,38 \%)$. Os medicamentos de referência e G2 apresentaram valores de $96,01 \%$ e $96,95 \%$ respectivamente, fato que torna similar a efetividade em seus diferentes processos produtivos, uma vez que a diferença entre os valores máximos e mínimos de teor de todos os medicamentos analisados é de apenas 3,56\%.

Para o teste de uniformidade de doses unitárias, a Farmacopeia Brasileira 5 $5^{\text {a }}$ d. (11) preconiza que comprimidos não revestidos que tenham a dose e proporção do fármaco com valor menor do que $25 \%$ de seu peso utilizem o método de Uniformidade de Conteúdo (UC), como é o caso da HCTZ $25 \mathrm{mg}$.
Novamente todos os comprimidos foram aprovados no teste, porém apresentaram resultados bem diferentes na escolha de seus casos, de acordo com a tabela de termos e expressões para o cálculo de valor de aceitação (VA) presente na Farmacopeia Brasileira $5^{\text {a }}$ Ed. (11). O medicamento $\mathrm{R}$ apresentou valor médio de teor em $96,49 \%$, sendo assim o seu caso de escolha $1 \mathrm{~b}$, pois apresenta valor médio inferior a $98,5 \%$. Realizado o cálculo do VA, obteve-se o resultado de 8,72, o qual cumpre o teste de UC.

Os medicamentos G1, G2 apresentaram valores médios de teor de respectivamente $100,17 \%$ e $101,30 \%$, os quais determinaram caso de escolha $1 \mathrm{a}(98,5 \%<\mathrm{X}<$ 101,5\%), com valores de aceitação 3,51 e 5,57; ambos os medicamentos cumpriram o teste. Por fim, o medicamento genérico $\mathrm{G} 3$, que apresentou valor médio de teor de $102,70 \%$, caso de escolha $1 \mathrm{c}(\mathrm{X}>101,5 \%)$, resultou em um VA de 6,64. Portanto, todos os medicamentos cumpriram o teste, uma vez que nenhum deles ultrapassou o valor de 15, que é o limite especificado pela Farmacopeia Brasileira 5 Ed. (11).

É de extrema importância terapêutica para os pacientes que teor e uniformidade de conteúdo do fármaco estejam dentro dos limites especificados, uma vez que quando estes se apresentam fora dos limites implicam diretamente na segurança proporcionada pelo medicamento, pois a inconstância de dose pode culminar em: i) superdosagem, podendo acarretar em toxicidade e aumento nos efeitos colaterais, e ii) subdosagem, podendo gerar falha terapêutica e, consequentemente, agravamento do quadro clínico apresentado pelo paciente.

Em relação ao teste de dissolução, para que os comprimidos de HCTZ sejam aprovados na etapa E1, devem alcançar não menos que $65 \%(\mathrm{Q}+5 \%)$ da quantidade declarada dissolvida após 30 minutos (Tabela 2). Novamente todos os produtos foram aprovados no teste, sendo o genérico G2 o medicamento com maior média de porcentagem dissolvida $(96,32 \%)$ e o medicamento de referência com o menor valor $(92,27 \%)$.

Os resultados podem ser considerados satisfatórios uma vez que a HCTZ é um fármaco com baixa hidrossolubilidade (16), e todos os laboratórios conseguiram ultrapassar em mais de $30 \%$ o valor de tolerância mínimo estabelecido pela Farmacopeia Brasileira $5^{\text {a }}$ Ed. (12), fato que demonstra certa equidade e possível efetividade terapêutica dos medicamentos, os quais devem ser melhor avaliados por testes posteriores de bioequivalência para que haja essa confirmação, ou seja, se após dissolvidos conseguem se manter nos níveis terapêuticos desejados. 
Por fim, o perfil de dissolução comparativo, representado pela Figura 3, avalia o fator de diferença entre os comprimidos e compara se estes têm ou não perfis de dissolução semelhantes.

É possivel observar que os comprimidos referentes às amostras G1 e G3 alcançaram valores de dissolução inferiores a $50 \%$ no intervalo de 5 minutos e valores inferiores a $75 \%$ no intervalo de 10 minutos, diferente das amostras $\mathrm{R}$ e $\mathrm{G} 2$, que superaram esses valores no primeiro intervalo e praticamente se igualaram no segundo intervalo.

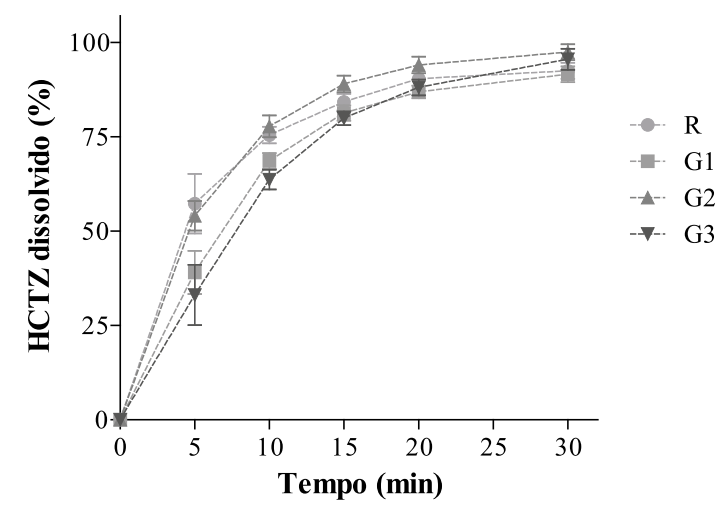

Figura 3. Perfis de dissolução da HCTZ a partir de comprimidos no estudo de perfil de dissolução comparativo entre medicamentos Referência (R) e Genéricos (G). $\mathrm{n}=12$; aparato I (cestas); $900 \mathrm{~mL} \mathrm{HCl}$ $0,1 \mathrm{M} ; 37 \pm 0,5^{\circ} \mathrm{C} ; 100 \mathrm{rpm} ; 0,5,10,15,20$ e $30 \mathrm{~min}$.

Esse fato pode ser novamente correlacionado ao teste de dureza e desintegração, em que os comprimidos G1 e G3 se apresentaram com valores mais elevados, podendo influenciar nas suas dissoluções, principalmente nos primeiros intervalos, uma vez que demoraram mais para se desintegrar e liberar seu conteúdo em meio de dissolução. As correlações apresentadas nas Figuras 4 e 5 ratificam esta hipótese, e demonstram como a dureza dos comprimidos afetou significativamente as quantidades de fármaco liberadas nos primeiros 5 e 10 minutos do ensaio, respectivamente.

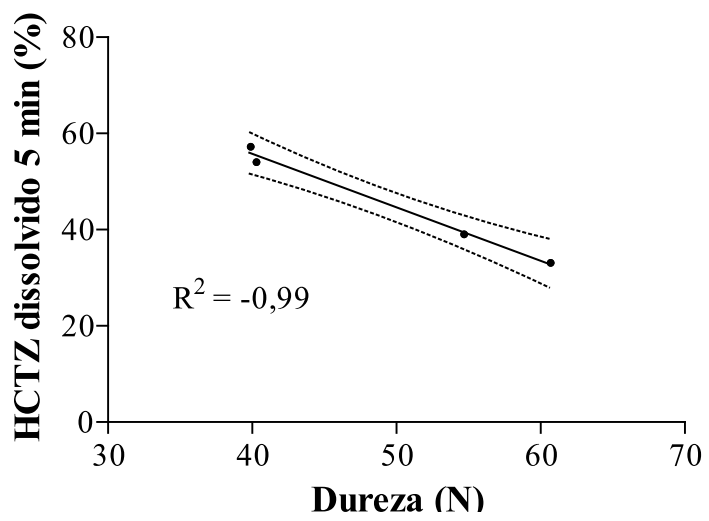

Figura 4. Correlação entre dureza e teor de HCTZ dissolvido em 5 minutos a partir de comprimidos Referência e Genéricos
Os parâmetros utilizados pela RDC 31/2010 (3) consideram que os medicamentos apresentam perfis de dissolução semelhantes quando alcançam valores F2 entre 50 e 100 .

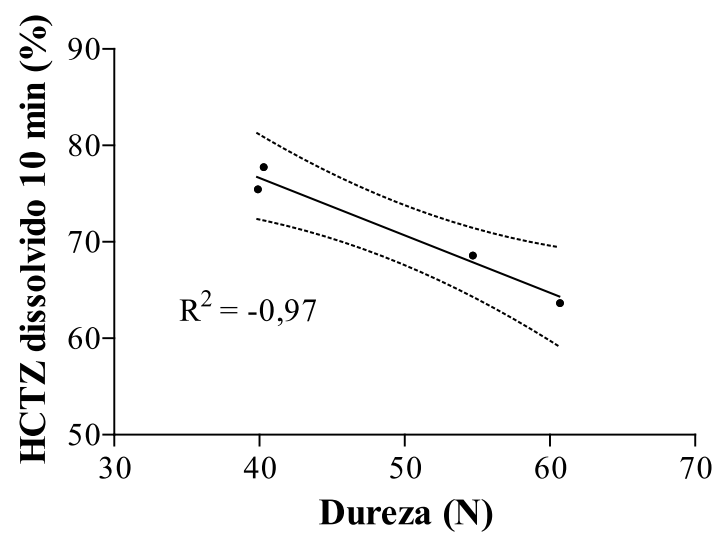

Figura 5. Correlação entre dureza e teor de HCTZ dissolvido em 10 minutos a partir de comprimidos Referência e Genéricos

Os medicamentos G1 e G2 quando comparados ao de referência tiveram um resultado para $\mathrm{F} 2$ de respectivamente 54,24 e 71,60, enquanto que G3 apresentou um valor de 47,39 conforme mostrado na Tabela 3 .

Sendo assim, pode ser considerado que os genéricos G1 e G2 apresentam perfis de dissolução semelhantes em relação ao medicamento $R$, enquanto que o genérico G3, não. Isto pode comprometer a bioequivalência e a intercambialidade entre o medicamento G3 e o medicamento $\mathrm{R}$, sobremaneira ao considerarmos que a HCTZ é um representante da Classe IV i.e., possui baixa solubilidade e baixa permeabilidade (16), de acordo com o Sistema de Classificação Biofarmacêutica (17). Portanto, a biodisponibilidade do fármaco pode ser ainda mais comprometida em função da disponibilização insuficiente ou inadequada.

Tabela 3. Fator de similaridade (F2) entre medicamentos Referência (R) e Genéricos ( $G$ ) contendo HCTZ (25 $\mathrm{mg}$ ) no estudo de perfil de dissolução comparativo

\begin{tabular}{cc} 
Medicamento & F2 \\
\hline $\mathrm{R} \times \mathrm{G} 1$ & 54,24 \\
$\mathrm{R} \times \mathrm{G} 2$ & 71,60 \\
$\mathrm{R} \times \mathrm{G} 3$ & $47,39^{*}$
\end{tabular}

$\mathrm{n}=12$; aparato I (cestas); $900 \mathrm{~mL} \mathrm{HCL} \mathrm{0,1M;} 37 \pm 0,5^{\circ} \mathrm{C} ; 100 \mathrm{rpm} ; 0$, 5, 10, 15, 20 e 30 min.; *Valor abaixo da especificação.

Outros estudos mostraram resultados semelhantes ao compararem medicamentos $\mathrm{R}$ com medicamentos $\mathrm{G}$ de HCTZ, além de similares. Mahle e cols (2007) compararam perfis de dissolução de comprimidos de HCTZ 
e obtiveram resultados dentro dos limites especificados para todos os medicamentos analisados, em que inclusive as amostras de medicamento similar apresentaram perfil de dissolução semelhante (18). Correia e cols (2015) em seu estudo também avaliaram o fator de semelhança F2 de comprimidos de HCTZ, disponíveis no mercado do Rio de Janeiro (19). Todos os genéricos analisados se enquadraram nos valores preconizados pela RDC 31/2010 (3), enquanto que os medicamentos similares não.

Sendo assim, os estudos refletem a qualidade de medicamentos Genéricos encontrados no mercado brasileiro e disponíveis no sistema público de saúde. O não cumprimento das normas estabelecidas pode impactar diretamente na qualidade de vida dos pacientes que utilizam estes medicamentos, em especial no caso da HCTZ, que se enquadra na classe de medicamentos de uso contínuo. Correia e cols (2015) ainda ressaltaram que o monitoramento constante por meio de estudos comparativos é uma medida que visa controlar a qualidade dos medicamentos disponíveis no mercado e que estes estudos podem apontar falhas no processo produtivo, resultando em possível ineficiência terapêutica (19).

\section{CONCLUSÃO}

Apenas dois dos três medicamentos genéricos testados (G1 e G2) apresentaram perfil de dissolução semelhante ao medicamento de referência. No entanto, não foram encontradas diferenças que podem impactar diretamente na saúde do paciente em relação aos outros testes realizados, pois todos os medicamentos alcançaram os valores preconizados pela Farmacopeia Brasileira $5^{\text {a }}$ ed. (2010). Em alguns casos, como por exemplo, no teste de doseamento e UDU, G2 e G3 mostraram resultados melhores que o próprio medicamento de Referência.

Pode-se, então, dizer que os resultados atingiram as expectativas, evidenciando qualidade por parte dos medicamentos Genéricos. A não semelhança no perfil de dissolução entre G3 e R serve de alerta para uma possível melhoria no processo produtivo desse medicamento, porém, não é possível condenar sua intercambialidade, tampouco sua eficácia terapêutica considerando apenas esse atributo de qualidade. O profissional farmacêutico tem importante papel nesse cenário de monitoramento da qualidade de medicamentos por meio de pesquisas científicas, e deve continuar desenvolvendo estudos que beneficiem o acesso a informação para a população, uma vez que é ele quem atua em contato direto com o paciente na orientação do uso de medicamentos, e presta os mais diversos serviços de assistência e cuidado farmacêutico.

\section{AGRADECIMENTOS}

Os autores agradecem o Laboratório de Análise de Medicamentos da Universidade Estadual de Londrina pelo suporte técnico disponibilizado na execução deste trabalho.

\section{REFERÊNCIAS}

1. BRASIL. Ministério da Saúde. Agência Nacional de Vigilância Sanitária. Lei $\mathrm{N}^{\circ} 9.787$, de 10 de fevereiro de 1999. Altera a Lei $n^{\circ} 6.360$, de 23 de setembro de 1976 , que dispõe sobre a vigilância sanitária, estabelece o medicamento genérico, dispõe sobre a utilização de nomes genéricos em produtos farmacêuticos e dá outras providências. Diário Oficial da República Federativa do Brasil, Poder Executivo, Brasília, DF, 10 fev. 1999.

2. Evaluate Pharma ${ }^{\circledR}$ World Preview 2015, Outlook to 2020. $8^{\text {th }}$ Ed. - June 2015. Disponível em: $<$ http://info. evaluategroup.com/rs/607-YGS-364/images/wp15.pdf $\geq$. Acesso em: 06 jun. 2016.

3. BRASIL. Ministério da Saúde. Agência Nacional de Vigilância Sanitária. Resolução de Diretoria Colegiada (RDC) $\mathrm{N}^{\mathrm{o}}$ 31, de 11 de agosto de 2010. Dispõe sobre a realização dos Estudos de Equivalência Farmacêutica e de Perfil de Dissolução Comparativo. Diário Oficial da União, Brasília, DF, 12 ago. 2010.

4. Odunfa OO, Adegoke OA, Onaga IC. Pharmaceutical Equivalence of Some Commercial Samples of Artesunate and Amodiaquine Tablets Sold in Southwestern Nigeria. Tropical J. Pharm. Res. 2009;8(6):491-499. DOI 10.4314/tjpr.v8i6.49392

5. Raw AS, Lionberger R, Yu LX. Pharmaceutical Equivalence by Design for Generic Drugs: Modified-Release Products. Pharm. Res. 2011;28:1445-1453. DOI: 10.1007/s11095-011-0397-6

6. Lourenço FR, Pinto TJA. Assessment of Pharmaceutical Equivalence: Difference Test or Equivalence Test? Lat. Am. J. Pharm. 2012;31(4):597-604. 
7. Adetunji OA, Adigun NF, Odeniyi MA. Pharmaceutical equivalent studies of some commercially available brands of Loratadine hydrochloride tablets. Afr J Med Med Sci. 2015;44(3):269-276.

8. Al Ameri MN, Nayuni N, Kumar KGA, Perrett D, Tucker A, Johnston A. The differences between the branded and generic medicines using solid dosage forms: In-vitro dissolution testing. Results Pharma Sci. 2012;2:1-8. DOI:10.1016/j.rinphs.2011.12.001

9. Storpirtis S, Marcolongo R, Gasparotto FS, Vilanova CMA. Equivalência farmacêutica no contexto da intercambialidade entre medicamentos genéricos e de referência: bases técnicas e científicas. Infarma 2004;16(910):51 - 56 .

10. Batlouni M. Diuréticos. Rev. Bras. Hipertens. 2009;16(4):211-214.

11. BRASIL. Ministério da Saúde. Agência Nacional de Vigilância Sanitária. Farmacopeia Brasileira 5 ed. Brasília: Fundação Oswaldo Cruz, 2010. v. 1

12. BRASIL. Ministério da Saúde. Agência Nacional de Vigilância Sanitária. Farmacopeia Brasileira 5 ed. Brasília: Fundação Oswaldo Cruz, 2010. v. 2

13. Lachman L, Lieberman HA, Kanig JL Teoria e prática na indústria farmacêutica. Lisboa: Fundação Calouste Gulbenkian, 2001.
14. Rowe RC, Sheskey PJ, Quinn ME. Handbook of Pharmaceutical Excipients. 6 ed. Pharmaceutical Press: London, 2009

15. Pessanha AFV, Rolim LA, Peixoto, MS, Silva RMF, Rolim Neto PJ. Influência dos excipientes multifuncionais no desempenho dos fármacos em formas farmacêuticas. Rev. Bras. Farm. 2012;93(2):136-145.

16. Ferreira TF, Mourão ASR, Ribeiro LAL, Freitas MB. Estudo comparativo da influência dos excipientes na qualidade de hidroclorotiazida $25 \mathrm{mg}$ em medicamentos referência e genéricos. Rev. Ciênc. Farm. Básica Apl. 2013;34(1):63-68.

17. Amidon GL, Lennernãs H, Shah VP, Crison JR. A theoretical basis for a Biopharmaceutic Drug Classification: The correlation of in vitro drug product dissolution and in vivo bioavailability. Pharm. Res. 1995;12(3):413-420.

18. Mahle F, Goelzer F, Adriano J, Felippe M, Vier N, Carli RBG, Rosa T, Couto AG, Lucinda-Silva R M. Avaliação do perfil de dissolução de comprimidos de hidroclorotiazida comercializados no Brasil. Rev. Ciênc. Farm. Básica Apl. 2007;28(3):265-271.

19. Correia LF, Gouvêa MM, Macedo EV, Peregrino CAF, Mourão SC. Avaliação da equivalência farmacêutica de comprimidos de hidroclorotiazida disponíveis no mercado do Rio de Janeiro. Rev Bras Farm. 2015;96(2):1266-1284. 